\title{
Anti-Nogo-A Antibody Treatment Enhances Sprouting of Corticospinal Axons Rostral to a Unilateral Cervical Spinal Cord Lesion in Adult Macaque Monkey
}

\author{
PATRICK FREUND, ${ }^{1 *}$ THIERRY WANNIER ${ }^{1,2 *}$ ERIC SCHMIDLIN, ${ }^{1 *}$ \\ JOCELYNE BLOCH, ${ }^{3}$ ANIS MIR, ${ }^{4}$ MARTIN E. SCHWAB, ${ }^{2}$ AND ERIC M. ROUILLER ${ }^{1}$ \\ ${ }^{1}$ Unit of Physiology and Program in Neurosciences, Department of Medicine, Faculty of \\ Sciences, University of Fribourg, CH-1700 Fribourg, Switzerland \\ ${ }^{2}$ Brain Research Institute, Department of Neuromorphology, University and ETH Zurich, \\ CH-8057 Zürich, Switzerland \\ ${ }^{3}$ Department of Neurosurgery, Neurosurgery Clinic, University Hospital of Lausanne, \\ CH-1011 Lausanne, Switzerland \\ ${ }^{4}$ Novartis Institute for Biomedical Research, CH-4002 Basel, Switzerland
}

\begin{abstract}
After injury, regrowth of axons in mammalian adult central nervous system is highly limited. However, in monkeys subjected to unilateral cervical lesion (C7-C8 level), neutralization of an important neurite outgrowth inhibitor, Nogo-A, stimulated axonal sprouting caudal to the lesion, accompanied by enhanced functional recovery of manual dexterity, compared with lesioned monkeys treated with a control antibody (Freund et al. [2006] Nat. Med. 12:790-792). The present study aimed at comparing the same two groups of monkeys for axonal sprouting rostral to the cervical lesion. The corticospinal tract was labeled by injecting the anterograde tracer biotinylated dextran amine into the contralesional motor cortex. The corticospinal axons were interrupted at the level of the lesion, accompanied by retrograde axonal degeneration (axon dieback), reflected by the presence of terminal retraction bulbs. The number of terminal retraction bulbs was lower in anti-Nogo-A antibody treated monkeys, and, when present, they were found closer to the lesion than in controlantibody treated monkeys. Compared with control antibody treated monkeys, the antiNogo-A antibody treated monkeys exhibited an increased cumulated axon arbor length and a higher number of axon arbors going in the medial direction from the white to the gray matter. Higher in the cervical cord (at C5 level), the anti-Nogo-A treatment enhanced the number of corticospinal fibers crossing the midline, suggesting axonal sprouting. Thus, the anti-Nogo-A antibody treatment enhanced axonal sprouting rostral to the cervical lesion; some of these fibers grew around the lesion and into the caudal spinal segments. These processes paralleled the observed improved functional recovery.
\end{abstract}

Indexing terms: spinal cord; anti-Nogo-A treatment; recovery; primate; lesion

\footnotetext{
The first three authors contributed equally to this work. Grant sponsor: Swiss National Science Foundation; Grant number: 31-61857.00; Grant number: 310000-110005 (to E.M.R.); Grant number: 31-63633 (to M.E.S.); Grant number: 4038043918/2 (PNR-38); Grant sponsor: Novartis Foundation; Grant sponsor: The Swiss National Science Foundation Centre of Competence in Research (NCCR) on "Neural plasticity and repair"; Grant sponsor: Christopher Reeves Foundation (Spinal Cord Consortium, Springfield, NJ).
}

*Correspondence to: Prof. Eric M. Rouiller, Unit of Physiology, Department of Medicine, University of Fribourg, Chemin du Musée 5, CH-1700 Fribourg, Switzerland. E-mail: eric.rouiller@unifr.ch 
After spinal cord injury, the regrowth of injured axons in the adult mammalian central nervous system (CNS) is extremely limited. Immediately after onset of injury, a series of structural changes occurs proximal and distal to the point of transection. Whereas the distal stump of the axon degenerates, corresponding to the so-called Wallerian degeneration, the proximal stump of the axon dies back (i.e., retracts) by several hundreds micrometers, as observed for example in the corticospinal (CS) tract of mice (Kerschensteiner et al., 2005). A typical feature of the transected axonal stumps proximal to the lesion is the formation of a terminal retraction bulb (Kalil and Schneider, 1975; Kao et al., 1977a,b; Houle and Jin, 2001).

The fate of axotomized CS neurons in the monkey is controversial, with claims of significant loss (see, e.g., Holmes and May, 1909; Wohlfarth, 1932; Levin and Bradford, 1938; Pernet and Hepp-Reymond, 1975), contrasting with evidence for survival of most CS neurons (Tower, 1940; Lassek, 1948). In a recent study, we confirmed that most CS neurons survived the axotomy, but their soma shrink (Wannier et al., 2005). In spite of the survival of their cell body, only a very limited regrowth of injured nerve fibers may take place in the adult mammalian CNS because of the presence of neurite outgrowth inhibitors, such as Nogo-A (see, e.g., Caroni et al., 1988; Schwab, 2004), within the CNS myelin. In fact, a therapeutic approach based on the application of antibodies neutralizing Nogo-A resulted in enhanced regrowth or regenerative sprouting of CS axons following spinal cord lesion in rats (see, e.g., Schnell and Schwab, 1990; Bregman et al., 1995; Thallmair et al., 1998; Brösamle et al., 2000; Liebscher et al., 2005; for review see Schwab, 2004), in marmosets (Fouad et al., 2004), and in macaque monkeys (Freund et al., 2006). Compared with animals also subjected to a spinal cord lesion and treated with a control antibody, the anti-Nogo-A antibody treatment promoted sprouting of CS axons caudal to the lesion, in the area of denervated motoneuron pools. In rats (Bregman et al., 1995; Thallmair et al., 1998; Schwab, 2004; Liebscher et al., 2005) and macaque monkeys (Freund et al., 2006), the sprouting of CS axons caudal to the lesion, promoted by anti-Nogo-A antibody treatment, was paralleled by an enhancement of functional motor recovery for various specific behavioral tasks, even leading to a complete recovery of performance on some of them.

Given that the regrowth of CS fibers caudal to a cervical spinal lesion occurred in parallel with better functional recovery of the hand in monkeys (Freund et al., 2006), the aim of the present study was to investigate, anatomically, how a cervical cord hemisection affects the CS axons rostral to the lesion in terms of dieback and formation of terminal retraction bulbs (see Fig. 1A). A specific goal was to assess whether the anti-Nogo-A antibody treatment may reduce or prevent axonal retraction effects proximal to the lesion and, in addition, favor local axonal sprouting. Thirteen macaque monkeys were therefore subjected to a cervical cord lesion unilaterally interrupting the dorsolateral funiculus; seven monkeys were treated with an antiNogo-A antibody, whereas the other six received a control antibody. The anterograde labeling of CS axons originating from the contralesional hemisphere, allowed us to address the following issues: 1) Does the proximal part of the axotomized CS axons retract from the lesion? If yes, in what proportion of CS axotomized axons does such a retraction occur and what is the distance of retraction? Does
anti-Nogo-A antibody treatment prevent such retraction of CS axons? 2) Do some axotomized CS axons attempt to reapproach and eventually enter the lesion? If yes, does the anti-Nogo-A antibody treatment make such attempts more successful? 3) Above the cervical lesion, do the axotomized CS axons sprout, giving rise to collaterals medially into the gray matter and/or growing around the lesion, thus possibly contributing to the enhanced presence of CS axons observed caudal to the lesion in anti-Nogo-A antibody treated monkeys (Freund et al., 2006)?

\section{MATERIALS AND METHODS Animals}

The experiments were conducted on 16 adult macaque monkeys (Macaca mulatta and $M$. fascicularis; see Tables 1, 2; see also Supplementary Table 1 in Freund et al., 2006) of either sex. Surgical procedures and animal care were conducted in accordance with the NIH Guide for the care and use of laboratory animals (ISBN 0-309-05377-3; 1996) and were approved by local (Swiss) veterinary authorities. Conditions for housing of the animals as well as the behavioral procedures aimed at assessing the manual dexterity of monkeys before and after the lesion were described in detail earlier (Schmidlin et al., 2004, 2005; Freund et al., 2006). Only the methods specifically relevant for the present study will be described below in detail. Among the 16 monkeys, 13 were subjected to a unilateral cervical cord lesion (see below). The lesioned monkeys were 3.5-6.9 years old at time of death, thus corresponding to young adult monkeys, and their weight ranged from 3.0 to $5.0 \mathrm{~kg}$. The other three monkeys (see Table 2 for age and weight) were intact and were considered here for comparison of some tracing data (see Fig. $5 \mathrm{E}, \mathrm{F})$ with the lesioned monkeys. One of the three intact monkeys was used in a previous tracing study describing the CS projection originating from the primary motor cortex (Rouiller et al., 1996).

\section{Surgical procedures: unilateral lesion of the dorsolateral funiculus}

In 13 monkeys, a unilateral cervical cord lesion was performed as follows. Intramuscular injection of ketamine (Ketalar; Parke-Davis; $5 \mathrm{mg} / \mathrm{kg}$, i.m.) was performed to induce anesthesia, and atropine was injected i.m. (0.05 $\mathrm{mg} / \mathrm{kg}$ ) to reduce bronchial secretions. Before surgery, the animal was treated with the analgesic Carprofen ( $\mathrm{Ry}$ madil, $4 \mathrm{mg} / \mathrm{kg}$, s.c.). Then, a continuous perfusion $(0.1$ $\mathrm{ml} / \mathrm{minute} / \mathrm{kg}$ ) through an intravenous catheter placed in the femoral vein with a mixture of $1 \%$ propofol (Fresenius) and a $4 \%$ glucose solution ( 1 volume of propofol and 2 volumes of glucose solution) induced a deep and stable anesthesia. The animal was then placed in a stereotaxic headholder, using ear bars covered at their tip with local anesthetic. Surgery was carried out under aseptic conditions. The following parameters were monitored: heart rate, respiration rate, expired $\mathrm{CO}_{2}$, arterial $\mathrm{O}_{2}$ saturation, and body temperature. In early experiments, an extra i.v. bolus of $0.5 \mathrm{mg}$ ketamine diluted in saline $(0.9 \%)$ was added at potentially more painful steps of the surgical procedure, such as laminectomy. In later experiments, ketamine was added to the perfusion solution and delivered throughout surgery $(0.0625 \mathrm{mg} / \mathrm{minute} / \mathrm{kg})$. With the animal placed in a ventral decubitus position, the spinal 
processes from $\mathrm{C} 2$ to Th1 were exposed. The paravertebral muscles were retracted, and the laminae of segments $\mathrm{C} 6$, C7, and Th1 were dissected. A complete C6 laminectomy and an upper $\mathrm{C} 7$ hemilaminectomy were then performed. The ligamentum flavum was removed in order to expose the dura mater, which was incised longitudinally. From previously available anatomical material, the rostrocaudal level at which the dorsal rootlets entered, respectively, cervical spinal segments 7 and 8 corresponded to the rostral zone of the spinal cord covered by cervical lamina 6 . The dorsal root entry zone at the C7/C8 border was then identified, providing a medial landmark for placing a surgical blade (No. 11; Paragon), which was used to perform an incomplete section of the cervical cord at this level. The surgical blade was inserted $4 \mathrm{~mm}$ in depth perpendicular to the spinal cord, and the section was prolonged laterally to completely cut the dorsolateral funiculus. In most cases, such a section completely interrupted the CS tract unilaterally. As illustrated in detail in a recent report (Freund et al., 2006), the lesion was aimed at a site caudal to the biceps motor nucleus but rostral to the nuclei of triceps, forearm, and intrinsic hand muscles (Jenny and Inukai, 1983). The muscles and the skin were sutured. The animal usually recovered from anesthesia 15-30 minutes after interruption of the perfusion with propofol and was treated postoperatively with an antibiotic (ampicilin 10\%, $30 \mathrm{mg} / \mathrm{kg}$, s.c.). During the week following the surgery, additional doses of Carprofen were given daily (pills of Rymadil mixed with food). After the spinal lesion, the animal was kept alone in a separate cage for a couple of days in order to perform a careful watch of its condition.

In addition, the transient separation in a cage allowed better conditions for recovery than the usual group housing with other monkeys, into which the animal was usually replaced 2-5 days after surgery. Some of the behavioral deficits resulting from the lesion were described previously (Freund et al., 2006) as well as the time course of recovery observed with various motor tasks.

\section{Anti-Nogo-A antibody treatment}

The treatment lasted for 4 weeks, using either the antiNogo-A antibody or control antibody ( $14.8 \mathrm{mg}$ in 4 weeks) delivered from an osmotic pump, placed in the back of the animal a few minutes after the lesion of the cervical cord. A small silastic tube, attached at one of its extremity to the pump, was positioned intrathecally $3-5 \mathrm{~mm}$ rostral to the cervical lesion. The pump had a volume of $2 \mathrm{ml}$, but one or the other of two types of pumps were used, allowing treatment during 4 or 2 weeks. In the latter case, after 2 weeks of treatment, the first pump was replaced under anesthesia by a second pump for another 2 weeks of treatment. Seven monkeys received either one of two monoclonal antibodies (mAbs) against different sites of Nogo-A (Table 1): the mouse mAB $11 \mathrm{C} 7$ (Liebscher et al., 2005) was raised against an 18-amino-acid peptide of rat Nogo-A (aa 623-640), close to the most inhibitory region of the Nogo-A protein (Oertle et al., 2003), which cross-reacts with mouse and monkey Nogo-A. The second antibody used, mAb hNogo-A, was raised by immunization with the whole Nogo-A-specific region of the human Nogo-A sequence. The characterization of the two anti-Nogo-A antibodies was performed as follows. Cynomologus monkey brain tissue (cerebral cortex) was homogenized in T-PER lysis buffer (Pierce, Rockford, IL) by using a rotor stator. For Western blots, aliquots corresponding to $10 \mu \mathrm{g}$ total protein were separated on a $4-12 \%$ NuPAGE gel (Invitrogen, Carlsbad, CA). The protein bands were transferred to a nitrocellulose membrane. The membrane was blocked for 1 hour at room temperature in blocking buffer [2\% blocking reagent (Amersham, Arlington Heights, IL) in TBS-T], then incubated with either $0.1 \mathrm{nM}$ hNogoA mAB or $1 \mathrm{nM} 11 \mathrm{C} 7$ antibody in blocking buffer for 2 hours, followed by 1 hour of incubation with either anti-human or anti-mouse peroxidase-coupled secondary antibodies (1: 500,000 dilution in blocking buffer). Signals were detected with ECL-Advance Western Blot detection reagents (Amersham) and exposure to film for 1 minute. Both antibodies recognize primate Nogo-A monospecifically on Western blots (Fig. 1B; see also Oertle et al., 2003). The antibodies were purified as IgGs and concentrated to $3-10 \mathrm{mg} / \mathrm{ml}$ in phosphate-buffered saline (PBS). In the other six monkeys, a control antibody was infused, corresponding to a purified IgG of a mouse $\mathrm{mAb}$ directed against wheat auxin (AMS Biotechnology, Oxon, United Kingdom).

In safety studies conducted in the monkey, remaining antibodies were retrieved from the pump at the end of the treatment (after 4 weeks), when the osmotic pumps were removed. The antibody $\mathrm{hNogoA} \mathrm{mAB}$ was found to be completely stable. Both anti-Nogo-A antibodies used were shown to be distributed with the flow of cerebrospinal fluid over most of the spinal cord and brain within 7 days of infusion and to penetrate deeply into the parenchyma (Weinmann et al., 2006). They are internalized together with endogenous Nogo-A protein into endosomal and lysosomal structures, leading to a down-regulation of Nogo-A (Weinmann et al., 2006). The concentrations chosen for the anti-Nogo-A antibody treatment $(3-10 \mathrm{mg} / \mathrm{ml}$ ) are high in relation to the high-subnanomolar affinities of the antibody for Nogo-A. Therefore, differences in efficacy are not expected at these concentrations. Because of the limited number of monkeys treated either with one (11C7) or the other (hNogoA) of the two anti-Nogo-A antibodies, the issue of whether there was a difference in efficacy between the two could not be addressed.

In the first six lesioned monkeys included in the study (pilot animals), the identity of the antibody contained in the pump was known to the experimenters (Supplementary Table 1 in Freund et al., 2006). For the seven other lesioned monkeys, the experimenters were blind to the antibody contained in the pump (Supplementary Table 1 in Freund et al., 2006) until the end of the experiment (killing of the animal and reconstruction of the lesion). The pump and the silastic tube were removed after four weeks of treatment. Each pump was then checked for the volume left to ensure that the antibody had been properly delivered.

\section{Tracing experiments and histological assessment of the lesion}

The anterograde tracer biotinylated dextran amine (BDA; Molecular Probes, Eugene, OR) was injected into the contralesional hemisphere (motor cortex) of the 13 lesioned monkeys to label the CS tract, using Hamilton syringes. Similarly, for comparison, BDA was injected unilaterally into the motor cortex of three additional intact monkeys (Table 2). Under propofol anesthesia (see above), a craniotomy was performed to expose the central and arcuate sulci. Injections of BDA were performed in the primary motor cortex (M1), i.e., in the rostral bank of the central sulcus, in a territory corresponding mainly to the 
TABLE 1. List of the Lesioned Monkeys Included in the Present Study With Identification Code ${ }^{1}$

\begin{tabular}{|c|c|c|c|c|c|c|c|c|c|}
\hline & $\mathrm{Mk}-\mathrm{CP}$ & Mk-CG & $\mathrm{Mk}-\mathrm{CB}$ & $\mathrm{Mk}-\mathrm{CH}$ & Mk-AP & Mk-AG & Mk-AM & Mk-AS & Mk-AC \\
\hline $\begin{array}{l}\text { Species } \\
\text { Anti-Nogo-A } \\
\text { treatment }\end{array}$ & $\begin{array}{l}\text { Fasc. } \\
\text { No }\end{array}$ & $\begin{array}{l}\text { Fasc. } \\
\text { No }\end{array}$ & $\begin{array}{l}\text { Fasc. } \\
\text { No }\end{array}$ & $\begin{array}{l}\text { Fasc. } \\
\text { No }\end{array}$ & $\begin{array}{c}\text { Fasc. } \\
\text { Yes } \\
(11 \mathrm{C} 7)\end{array}$ & $\begin{array}{c}\text { Fasc. } \\
\text { Yes } \\
\text { (hNogoA) }\end{array}$ & $\begin{array}{c}\text { Fasc. } \\
\text { Yes } \\
\text { (hNogoA) }\end{array}$ & $\begin{array}{l}\text { Mul. } \\
\text { Yes } \\
(11 \mathrm{C} 7)\end{array}$ & $\begin{array}{c}\text { Fasc. } \\
\text { Yes } \\
(\text { hNogoA) }\end{array}$ \\
\hline "Double-blind" procedure & Yes & Yes & Yes & Yes & Yes & No & Yes & No & Yes \\
\hline Completeness of CS (dlf) section & $\begin{array}{c}\text { No } \\
\text { (BDA) }\end{array}$ & $\begin{array}{c}\text { Yes } \\
(\mathrm{BDA})\end{array}$ & $\begin{array}{c}\text { Yes } \\
(\mathrm{BDA})\end{array}$ & $\begin{array}{c}\text { Yes } \\
(\mathrm{BDA})\end{array}$ & $\begin{array}{c}\text { Yes } \\
(\mathrm{BDA})\end{array}$ & $\begin{array}{c}\text { Yes } \\
(\mathrm{BDA})\end{array}$ & $\begin{array}{c}\text { Yes } \\
(\mathrm{BDA})\end{array}$ & $\begin{array}{c}\text { No } \\
\text { (BDA) }\end{array}$ & $\begin{array}{c}\text { Yes } \\
(\mathrm{BDA})\end{array}$ \\
\hline Extent of hemicord lesion (\%) & 45 & 51 & 75 & 90 & 58 & 78 & 80 & 41 & 85 \\
\hline Extent of gray matter cut (\%) & 77 & 73 & 81 & 74 & 85 & 81 & 71 & 86 & 100 \\
\hline Number of BDA-labeled CS axons at C5 & 927 & 1,005 & 1,186 & 780 & 1,250 & 2,282 & 1,148 & 168 & 270 \\
\hline Percentage of uncrossed CS axons at C5 & 10 & 5 & 9 & 3 & 5 & 9 & 5 & 11 & 7 \\
\hline Volume of BDA injected (in $\mu \mathrm{l}$ ) & 24 & 24 & 20 & 20 & 24 & 28 & 19 & 22 & 20 \\
\hline No. of BDA injection sites & 12 & 12 & 10 & 10 & 12 & 15 & 10 & 11 & 10 \\
\hline Total No. of injection tracks & 6 & 6 & 5 & 5 & 6 & 7 & 6 & 4 & 6 \\
\hline Survival time post-BDA injection (days) & 78 & 70 & 78 & 62 & 81 & 70 & 69 & 72 & 64 \\
\hline No. of days between lesion and BDA injection & 81 & 70 & 147 & 76 & 79 & 42 & 69 & 210 & 71 \\
\hline Age of the animal at sacrifice (years) & 6.9 & $\sim 4$ & 5 & $\sim 4$ & 6.5 & 3.5 & $\sim 4$ & 6.25 & $\sim 4$ \\
\hline 1) No. of retraction bulbs/No. of CS axons at C5 & 0.06 & 0.053 & 0.116 & 0.09 & 0.058 & 0.019 & 0.004 & 0.006 & 0.015 \\
\hline No. of CS axons with terminal bulbs & 56 & 53 & 137 & 70 & 72 & 44 & 5 & 2 & 4 \\
\hline $\begin{array}{l}\text { 2) Mean distance of axonal retraction in } \mu \mathrm{m} \\
\text { (SD) }\end{array}$ & $\begin{array}{l}291.5 \\
(98.3)\end{array}$ & $\begin{array}{c}326 \\
(292.3)\end{array}$ & $\begin{array}{c}407.5 \\
(171.2)\end{array}$ & $\begin{array}{c}310.7 \\
(154.5)\end{array}$ & $\begin{array}{c}240.8 \\
(144.5)\end{array}$ & $\begin{array}{c}145.9 \\
(101.8)\end{array}$ & $\begin{array}{c}116.5 \\
(155.3)\end{array}$ & - & $\begin{array}{c}179.4 \\
(143.8)\end{array}$ \\
\hline $\begin{array}{l}\text { Percentage of terminal retraction bulbs } 100 \mu \mathrm{m} \\
\text { rostral to the lesion }\end{array}$ & 13 & 7 & 1 & 7 & 27 & 36 & 60 & 0 & 40 \\
\hline $\begin{array}{l}\text { 3) Normalized axon arbor length rostral to } \\
\text { lesion }\end{array}$ & 41.624 & 14.961 & 35.769 & 23.33 & 41.914 & 71.12 & 64.752 & - & 31.307 \\
\hline $\begin{array}{l}\text { 4) Normalized No. of CS axotomized axons } \\
\text { entering lesion area }\end{array}$ & 0.003 & 0.0039 & 0.0093 & 0.014 & 0.03 & 0.022 & 0.0366 & - & 0.0851 \\
\hline $\begin{array}{l}\text { 5) Normalized No. of CS axons going from } \\
\text { white to gray matter }\end{array}$ & 0.135 & - & 0.111 & 0.128 & 0.213 & 0.301 & 0.169 & - & - \\
\hline $\begin{array}{l}\text { 6) Normalized No. of CS axons crossing midline } \\
\text { at C5 }\end{array}$ & 0.045 & 0.028 & 0.025 & 0.041 & 0.06 & 0.06 & 0.05 & - & - \\
\hline
\end{tabular}

${ }^{1}$ Detailed information on the nine monkeys included in the present anatomical study. Eight of the nine monkeys were part of the previous report, describing the behavioral recovery and the properties of the CS axon collaterals caudal to the lesion (Freund et al., 2006; see their Table 1). One additional animal (Mk-AG) was newly introduced in the present study. At the time of the experiment, the monkeys had different names, not indicating whether the animal was infused with the control or the anti-Nogo-A antibody. Under species, Mul. is for Macaca mulata and Fasc. is for Macaca fascicularis.The four control-antibody-treated monkeys (Anti-Nogo-A treatment: No) are in the four leftmost columns, whereas the is for Macaca mulata and Fasc. is for Macaca fascicularis. The four control-antibody-treated monkeys (Anti-Nogo-A treatment: No) are in the four leftmost columns, whereas the $\mathrm{mAB}$ hNogoA). Under Double-blind procedure, "yes" refers to monkeys for which the collaborators delivering the antibodies (control or anti-Nogo-A) did not know to which monkey they were aimed for. On their side, the experimenters taking care of the monkeys did not know which antibody has been administered to the corresponding animal. In the row Completeness of CS (dlf) section, "yes" and "no" indicate whether the dorsolateral funiculus (dlf) was or was not completely transected unilaterally, respectively. BDA in parentheses means that completeness of the section of the dorsolateral funiculus unilaterally was assessed based on the BDA labeling of the CS tract immediately above the lesion. If yes, this means that there was no BDA-labeled axon spared by the lesion in the aimed dorsolateral funiculus. If no, this means that few BDA-labeled axons in the dorsolateral funiculus were spared by the lesion. For four animals, their age was not precisely determined when they were received in our animal facility and was estimated to be about 3 years at that time. Therefore, their age was estimated to be about 4 years old at death. Dashes are for parameters that could not be determined in the corresponding monkeys.

hand representation. Based on previously available monkeys in which the hand representation was determined by intracortical microstimulation (Rouiller et al., 1996, 1998; Schmidlin et al., 2004, 2005), the hand territory was estimated to be located immediately rostral to the central sulcus, extending mediolaterally between 10 and $15 \mathrm{~mm}$ from the midline, with its most lateral extent corresponding roughly to the genu of the arcuate sulcus. In this territory of about 5-6 $\mathrm{mm}$ along the mediolateral axis, three to four syringe penetrations were aimed perpendicularly to the cortical surface, at 1.5-2 $\mathrm{mm}$ distance from each other. To cover most of the rostral bank of the central sulcus, BDA was typically deposited along each syringe penetration at two (rarely three) depths, usually at sites located 3 and $7 \mathrm{~mm}$ below the pial surface. Usually one to three additional syringe penetrations were performed more medially, still along the central sulcus, to cover the representation of more proximal territories (wrist, elbow, shoulder, trunk). The detailed parameters of BDA injections are given for each monkey in Table 1. Based on our previous experience with tracing the CS tract with BDA in monkeys (Rouiller et al., 1996), the survival time after BDA injection was set to 3 weeks for the first two lesioned animals. However, it turned out that the cervical lesion substantially slowed the anterograde axonal transport of BDA, so the tracer did not reach the cervical segments of interest. For this reason, a much longer survival time (around 60-80 days) was applied to the other 11 lesioned monkeys, allowing transport of BDA up to the thoracic level. Furthermore, it appeared that, in two lesioned monkeys, the BDA staining was not dense enough in the cervical cord for a full anatomical analysis. Consequently, these four animals (too short survival time or insufficient staining) were not considered further in the present study. The list of the nine lesioned monkeys considered in the present study for the analysis of CS axons rostral to the cervical lesion is given in Table 1.

At the end of the survival period, the animals were killed under deep (lethal) anaesthesia (90 mg sodium pentobarbital/kg body weight) by transcardiac perfusion with $0.9 \%$ saline $(400 \mathrm{ml})$. The perfusion was continued with fixative (3 liters of $4 \%$ phosphate-buffered paraformaldehyde in $0.1 \mathrm{M}$ phosphate buffer, $\mathrm{pH}$ 7.6) and solutions (2 liters each) of the same fixative containing increasing concentrations of sucrose $(10 \%, 20 \%$, and $30 \%)$. The brain and spinal cord were dissected and placed in a $30 \%$ solution of sucrose (in phosphate buffer) for cryoprotection for 7 days. Frozen sections ( $50 \mu \mathrm{m}$ thick) of the brain were cut in the frontal plane, whereas frozen sections (50 $\mathrm{mm}$ thick) of the cervical cord (approximately segments C6-T3) were cut in the parasagittal longitudinal plane and collected in three series for later histological processing. Upper cervical segments and lower thoracic spinal segments were cut in the frontal plane at $50 \mu \mathrm{m}$ thick, and sections were also collected in three series. BDA staining was revealed in one series of spinal cord sections, as described in detail in previous reports (Rouiller et al., 1996, 1998). The second series of spinal cord sections was 
immunohistochemically processed to visualize corticospinal axons by using the marker SMI-32, as recently reported (Wannier et al., 2005), a series of sections also used to reconstruct the location and extent of the cervical lesion (Fig. 1C), as described in detail earlier (Schmidlin et al., 2004; Wannier et al., 2005). The third series of sections was processed to visualize another anterograde tracer (dextran-fluorescein) injected into the ipsilesional hemisphere, but these data will not be presented here. A light microscope (Olympus) and Neurolucida software were used for analyses of CS axon numbers, retraction, arbor length, sprouting, and trajectories.

\section{Normalization of BDA-labeled CS axons}

Because of variations in the uptake of BDA (Table 1; see also Supplementary Table 1 in Freund et al., 2006), the number of BDA-labeled CS axons varied from one animal to the next. To normalize the BDA data across monkeys, the total number of BDA-labeled CS axons was counted at the C5 level on a frontal section in the white matter, in the two dorsolateral funiculi, and in the ventral funiculus ipsilateral to the BDA injection in the cerebral cortex (Table 1).

\section{Measurement of retraction of lesioned CS axons and number of CS axons with terminal retraction bulbs}

On the photomicrographs of the parasagittal cervical cord sections processed for BDA visualization, a first line was drawn along the rostral border of the lesion in Corel Draw. A second line, parallel to the first one, was drawn $600 \mu \mathrm{m}$ more rostrally, following the orientation of the axons. The first line offered a fixed point of reference for measuring the distance of CS axonal retraction (Fig. 2A). The distance of the center of each terminal bulb (identified as being at least twice the axon caliber) to the lesion border was measured within this space. Only CS axons with terminal bulbs located in the white matter at a maximal distance of $600 \mu \mathrm{m}$ from the edge of the lesion were used for this analysis (Fig. 2A). A more specific question was addressed to the terminal bulbs located in the first $100 \mu \mathrm{m}$ from the rostral border of the lesion. CS axon arbors displaying no such terminal bulbs were not taken into consideration for measurement, because it could not be excluded that the cut end of the axons was leaving the plane of the section, thereby continuing on an adjacent section. The cumulative number of CS axon with terminal bulbs observed on the BDA sections analyzed was finally divided by the total number of BDA-labeled CS axons observed at C5, for normalization as explained above. These assessments of the number of CS axons with terminal retraction bulbs (Fig. 2D,F) and the distance of retraction (Fig. 2E) are represented schematically by the numbers 1 and 2 in Figure 1A.

\section{Measurement of CS axon arborization and CS sprouting}

In the gray matter, the presence of BDA-labeled CS axon arbors was investigated at the immediate border of the lesion and within an area extending up to $500 \mu \mathrm{m}$ away from the rostral limit of the lesion at a total magnification of $\times 200$. For this analysis (schematically represented by the number 3 in Fig. 1A), all parasagittal spinal cord sections processed for BDA visualization $(150 \mu \mathrm{m}$ intervals) on the ipsilesional cervical side were chosen. In Neurolucida software, each BDA-labeled axon segment in the gray matter was traced. Then, the cumulated axon arbor length was computed and normalized with respect to the total number of CS axons labeled at C5 level, as described above.

By using Neurolucida as well, the BDA-labeled CS axon arbors which attempted to enter the lesion by crossing the rostral limit of the lesion were drawn. The accumulated number of CS axon arbors intercepting the rostal border of the lesion was determined in each monkey (analysis represented schematically by the number 4 in Fig. 1A).

To analyze further the sprouting of the CS axons in the region rostral to the lesion, BDA-labeled CS axon arbors entering into the gray matter were counted up to a distance of $3 \mathrm{~mm}$ away from the rostral border of the lesion on the parasagittal sections (number 5 in Fig. 1A). For this analysis, histological sections exhibiting BDA-stained CS axons in the white and gray matter were selected (ranging from five to nine slides across animals). At their crossing with the white/gray matter border, these axon arbors were generally oriented along a perpendicular axis, although some of them showed an orientation that was slightly oblique from the axis perpendicular to the white/gray matter border.

Furthermore, at more rostral levels in the cervical cord, five frontal sections taken at C5 level were selected to count the number of CS fibers crossing the midline at C5 (analysis represented schematically by the number 6 in Fig. 1A). In Neurolucida, a vertical line was superimposed onto the midline of the frontal section of the cervical cord at C5 level, and two additional vertical lines were drawn at a distance of $250 \mu \mathrm{m}$ on each side away from the line on the midline (see Fig. 5C). CS axon arbors crossing these lines were counted (if the same axon arbor was long enough to intercept two or three lines, it was counted once) and the cumulative number for the five frontal sections was normalized by dividing by the total number of CS axons present at C5 level in the white matter, as described above.

For illustrations (see Figs. 2, 3, 5), photomicrographs were captured from an Olympus microscope (BX40) in Neurolucida and then stored as computer files for final processing in Corel Draw 12 or Corel Draw X3 software for production of the final montage. Contrast and brightness adjustments were performed in Adobe ImageReady CS.

\section{RESULTS \\ Morphology of the unilateral cervical cord lesion}

After unilateral injection of BDA into the contralesional primary motor cortex (M1) mainly in the hand representation, the main CS tract was found at cervical cord level C5 in the left dorsolateral funiculus, representing 90-95\% of the whole CS population (Fig. 1A). The uncrossed CS projection (representing 5-10\% of CS axons) was found in the dorsolateral and ventral funiculi homolateral to the BDA injection (Fig. 1A). These figures are consistent with a recent report on the distribution of CS axons at the level of the lumbar cord (Lacroix et al., 2004). The unilateral section of the cervical cord was aimed at transecting the crossed CS tract in the left dorsolateral funiculus (Fig. $1 \mathrm{C})$. In seven of nine lesioned monkeys included in the 

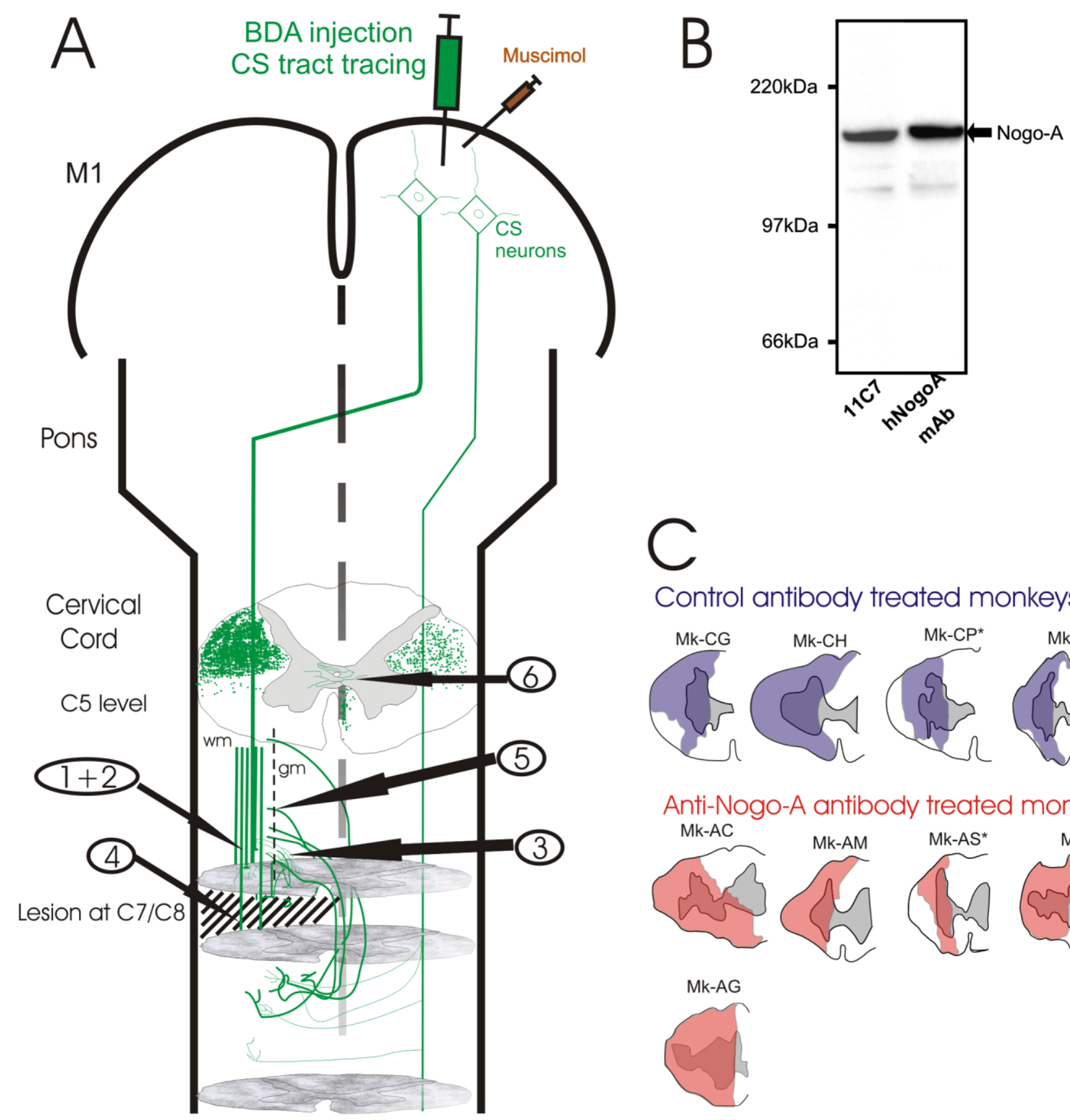

Fig. 1. A: Schematic representation of the macaque monkey corticospinal (CS) projection originating from the right primary motor cortex (M1) as seen at the cervical cord level, as a result of BDA injection (green syringe) in the right motor cortex. At the C5 level, on a frontal section of the cervical cord, green dots indicate the location and distribution of the CS axons, forming the dorsolateral funiculus (dlf) contralateral to the injection site, and the uncrossed CS axons, present in the dorsolateral and ventral funiculi ipsilateral to the injection site. At the C7/C 8 level, the ensemble of black oblique lines represents the cervical lesion performed in the present study in 13 monkeys. Rostral to the lesion, parallel thick green lines represent CS axons in the dorsolateral funiculus, interrupted by the lesion. Axonal sprouting is represented by thinner lines arising from the thick green lines. The numbers $1-6$ point to the six sites of the quantitative analyses performed in the present study as explained in Materials and Methods. The brown syringe is for pharmacological reversible inactivation experiments of the contralesional primary motor cortex (M1), as previously reported (Schmidlin et al., 2004) and mentioned in

the Discussion. The thick vertical dashed line represents the midline, whereas the thin vertical dashed line represents the boundary between the white (wm) and the gray (gm) matter. A part of this schematic has been published previously (Freund et al., 2006). B: Both monoclonal antibodies against Nogo-A, $11 \mathrm{C} 7$ and antihNogo-A, recognize a single band running at the expected molecular weight of Nogo-A in cortex homogenates of adult macaque monkeys separated by SDS-polyacrylamide gel electrophoresis. C: Reconstruction from parasagittal sections in the frontal plane of the location and extent of the lesion at C7/C8 lesion performed in the nine monkeys included for the present analysis of the CS tract rostral to the lesion. Lesions in blue are for the control antibody treated monkeys $(n=4)$, whereas red represents the lesion in the anti-Nogo-A antibody treated monkeys $(n=5)$. The asterisks indicate the two monkeys with incomplete lesion of the dorsolateral funiculus (see text). Among the nine lesion reconstructions, eight have been previously shown by Freund et al. (2006). 

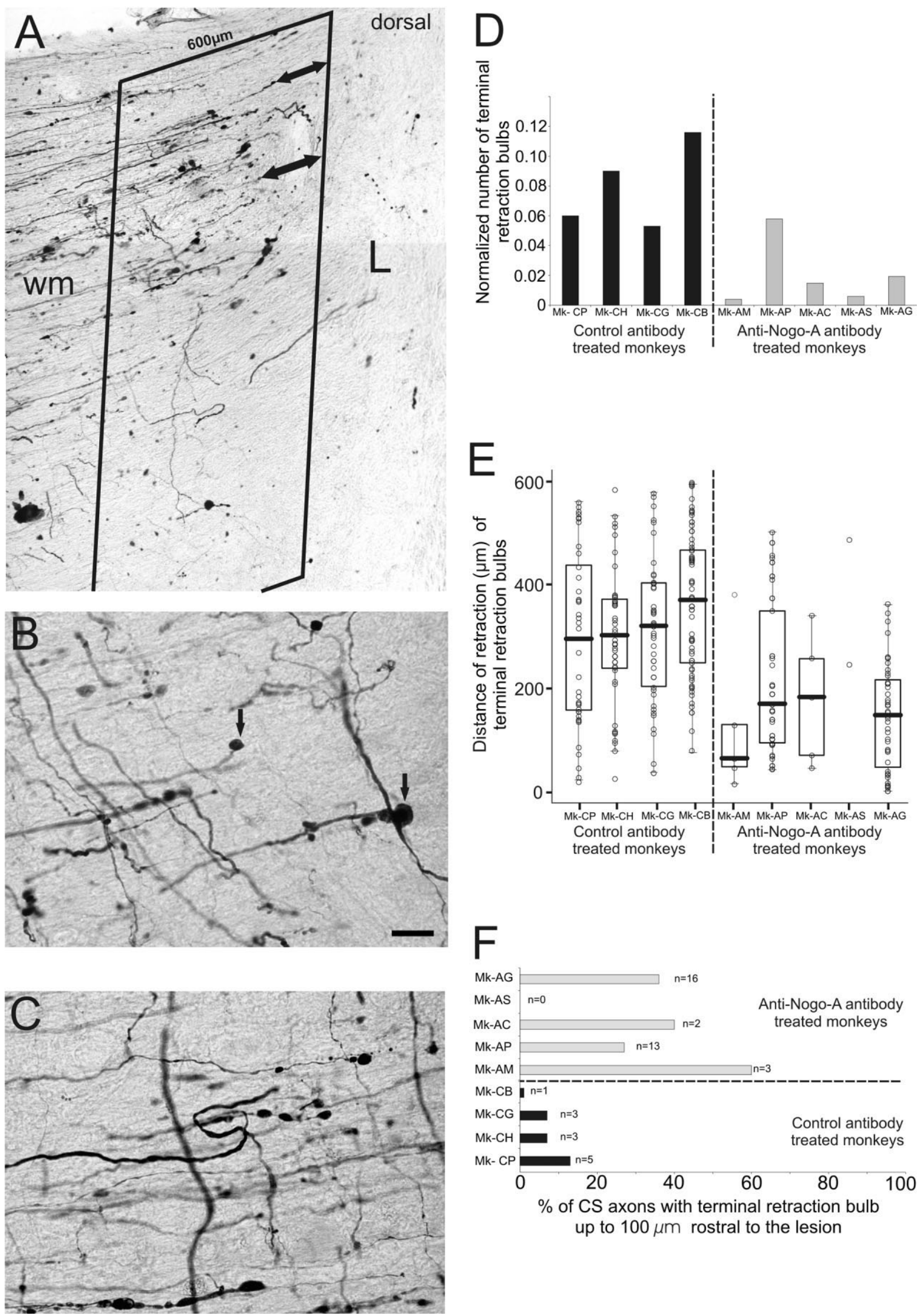
present anatomical analysis, the dorsolateral funiculus was indeed completely interrupted, as assessed on the basis of the location and extent of the lesion (Fig. 1C) in comparison with the location and extent of the dorsolateral funiculus in an intact monkey after BDA injection in M1. In addition, BDA labeling of CS axons in the white matter provided evidence that all axons in the dorsolateral funiculus were indeed sectioned in these seven monkeys. On the other hand, in two monkeys (indicated by an asterisk in Fig. 1C), the lesion did not completely interrupt the dorsolateral funiculus in its most ventrolateral extent, so some CS fibers were spared. The intact BDAlabeled CS fibers were easily detectable because of their straight course in the white matter; these were excluded from the anatomical analysis. The lesion territory was easily distinguishable from the intact spinal cord tissue. A scar consisting of nonhomogenous tissue and of cysts of various diameters was observed, acting as a clear physical impediment for axonal regrowth in the rostrocaudal direction (Fig. 3A).

\section{Effects of anti-Nogo-A antibody treatment on $\mathrm{CS}$ axons in the region rostral to the cervical cord lesion}

To evaluate whether the anti-Nogo-A antibody treatment favored the maintenance, growth, and/or sprouting from the CS axons in the area rostral to the cervical cord lesion, six quantitative morphological analyses were conducted at specific locations, represented schematically by the numbers 1-6 in Figure 1A and presented below in the following six sections. The detailed results obtained for these six analyses are given for each individual lesioned monkey in Table 1 .

Appearance and number of terminal retraction bulbs on transected BDA labeled CS axons. In all monkeys subjected to cervical cord lesion, BDA-labeled CS axons displaying terminal retraction bulbs were found rostral to the lesion (Fig. 2A-C), corresponding to a common feature for transected axons that underwent retrograde degeneration (Kalil and Schneider, 1975; Kao et al., 1977a,b; Houle and Jin, 2001). To investigate the influence of anti-Nogo-A antibody treatment on the transected CS axons, the region rostral to the lesion was scanned at high magnification for CS axons exhibiting terminal retraction bulbs.

Terminal retraction bulbs in the area rostral to the lesion, in a territory of white matter extending $600 \mu \mathrm{m}$ away from the lesion border, were counted in each monkey and accumulated for all parasagittal sections analyzed.
For normalization, the accumulated numbers of terminal retraction bulbs in each monkey were divided by the total number of BDA-labeled CS fibers in the white matter at C5 level. Strikingly, in anti-Nogo-A antibody treated monkeys, the normalized cumulated numbers of CS axons exhibiting terminal retraction bulbs were significantly lower (approximately fourfold on average) than in control antibody treated monkeys (Fig. 2D). This difference was statistically significant (Mann and Whitney test, $P<$ 0.05). Furthermore, from a qualitative point of view, antiNogo-A antibody treated monkeys, instead of exhibiting the standard terminal retraction bulbs, often showed outgrowing sprouts being tortuous in shape and decorated with varicosities, strongly resembling boutons en passant and terminaux that approached the lesion border (Fig. $3 \mathrm{~B}, \mathrm{C})$.

CS axonal retraction (axonal dieback). To address the issue of a response of the transected CS axons to anti-Nogo-A antibody treatment, the distance of retraction from the lesion for the CS axons exhibiting a terminal retraction bulb was measured. All terminal retraction bulbs within a distance of $600 \mu \mathrm{m}$ from the rostral limit of the lesion were included.

The average retraction distance from the lesion border was markedly reduced in anti-Nogo-A antibody treated monkeys compared with the control antibody treated monkeys (Fig. 2E). This difference was not statistically significant (Mann and Whitney test, $P>0.05$ ). However, because of the large variability across monkeys and because of the much lower number of CS axons with terminal retraction bulbs in the anti-Nogo-A antibody treated monkeys with regard to that in the control antibody treated monkeys, the conditions were not favorable for detecting a possible treatment-induced population difference (Fig. $2 \mathrm{E}$ ).

A prominent difference appeared, however, when we focused on a more restricted area, within $100 \mu \mathrm{m}$ of the rostral limit of the lesion (Fig. 2F). About $10 \%$ of transected CS axons displaying a terminal retraction bulb were found in the control antibody treated monkeys. In contrast, this proportion ranged between $27 \%$ and $60 \%$ in the anti-Nogo-A antibody treated monkeys (see also Table 1). Insofar as the total number of terminal retraction bulbs was much higher in the control antibody treated monkeys (Fig. 2D), the data in Figure 2F indicate that the transected CS axons in anti-Nogo-A antibody treated monkeys rarely formed terminal retraction bulbs, but, when they did, they were located close to the lesion. In contrast, the numerous terminal retraction bulbs in the
Fig. 2. A-C: A displays transected BDA-labeled CS fibers at the proximity of the rostral border of the lesion in the white matter. The lesion border appears as nonhomogenous axon-free tissue distinct from more rostral intact spinal cord tissue. The arrows with double heads illustrate how the distance was measured between a terminal retraction bulb and the rostal border of the lesion. L, lesion; wm, white matter. $\mathrm{B}$ and $\mathrm{C}$ illustrate at higher magnification BDA-labeled CS axons close to the lesion forming retraction bulbs (arrows). D: Normalized number of terminal retraction bulbs observed within a territory extending $600 \mu \mathrm{m}$ away from the lesion border in the rostral direction for the nine lesioned monkeys included in the present study, separately for the control antibody treated monkeys $(n=4)$ and the anti-Nogo-A antibody treated monkeys $(n=5)$. The site of this analysis is schematically represented by the number 1 in Figure 1A. E: For each monkey, the distance between each terminal retraction bulb observed and the rostal limit of the lesion was plotted. Superimposed on the individual measurements, the box and whisker plot depicts the median value (horizontal bar in the middle of the box) and the 25th and 75 th percentiles (bottom and top extremities of the box, respectively). The site of this analysis is schematically represented by the number 2 in Figure 1A. F: Instead of considering a territory of $600 \mu \mathrm{m}$ away from the lesion in the rostral direction (A,D,E), a more restricted area only $100 \mu \mathrm{m}$ away from the lesion was considered. The horizontal bar represents the percentage of the total number of terminal retraction bulbs observed up to $600 \mu \mathrm{m}$ away from the lesion, which terminates not farther than $100 \mu \mathrm{m}$ from the rostral border of the lesion. Scale bars $=50 \mu \mathrm{m}$. 

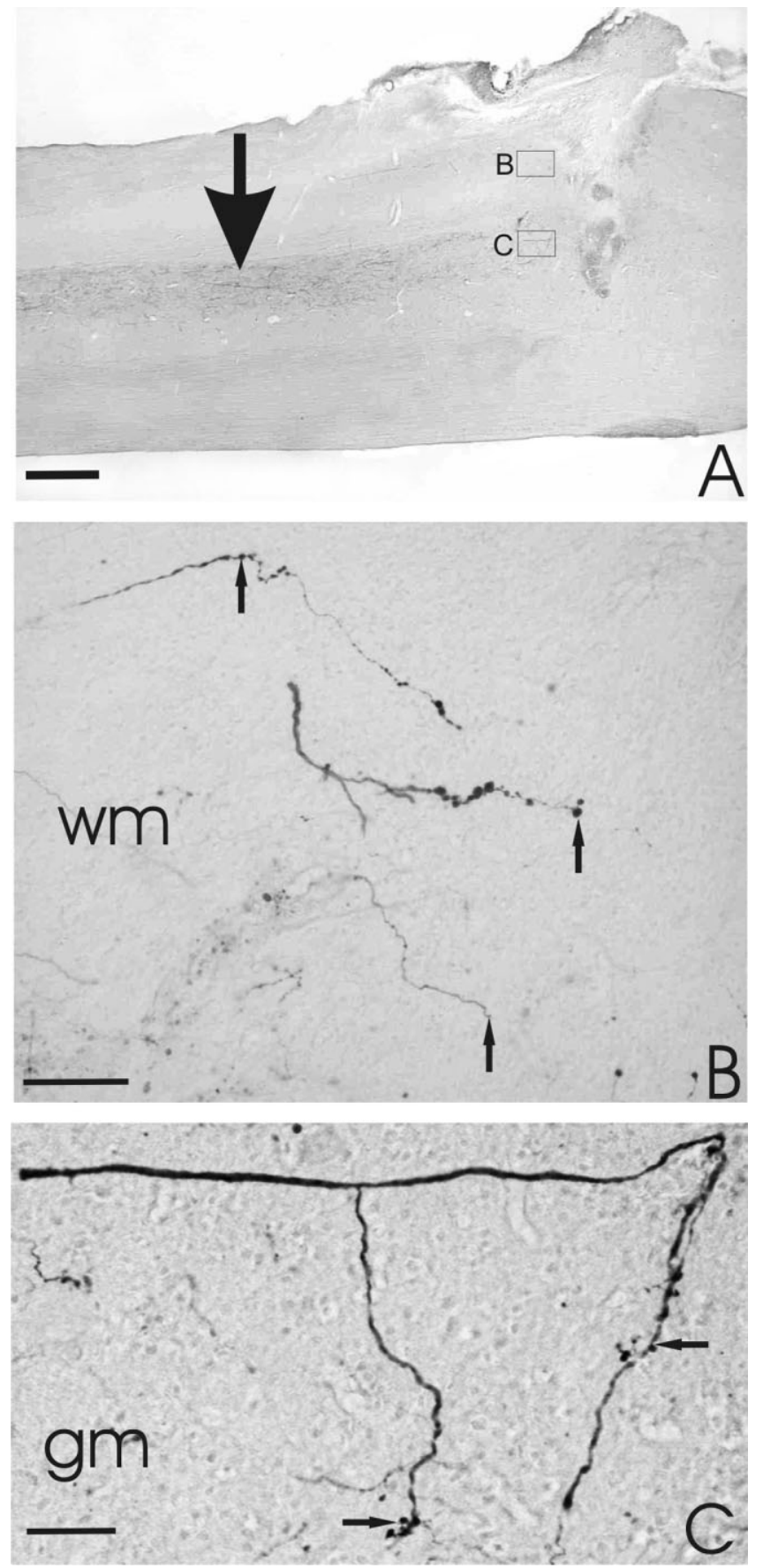

Fig. 3. A: Photomicrograph of a parasagittal spinal cervicalthoracic cord section illustrating the appearance of BDA-labeled CS fibers in the gray matter, rostral to the lesion (arrow). The section was taken from an anti-Nogo-A antibody treated monkey. The CS axons in the white matter and the CS axon arbors in the gray matter, shown at higher magnification in $\mathrm{B}$ and $\mathrm{C}$, are located in the cervical cord as indicated by the two rectangles. B,C: Typical examples of sprouting from transected CS axons in the white matter (B) and gray matter (C) giving rise to collaterals directed toward the lesion border. These axons were tortuous and irregular in growth, with varying diameter and varicosities (arrows) along their course and at their termination. wm, White matter; gm, gray matter. Scale bars $=1 \mathrm{~mm}$ in A; $100 \mu \mathrm{m}$ in $\mathrm{B} ; 50 \mu \mathrm{m}$ in $\mathrm{C}$. control antibody treated monkeys had generally retracted farther from the lesion.

Density of CS axon arbors in the gray matter rostral to the lesion. To investigate whether anti-Nogo-A antibody treatment induced changes within the gray matter of the cervical cord above the lesion, the BDA-labeled CS axon arbors within the spinal cord $500 \mu \mathrm{m}$ rostral to the lesion on parasagittal sections were traced bilaterally at a magnification of $\times 200$. The sections were then superimposed (Fig. 4A-D), showing for each animal the density of axon arbors in the white matter rostral to the lesion (brown lines) as well as in the gray matter rostral to the lesion (black lines on the left side of the lesion shown in Fig. 4A-D). For completeness of the information, BDAlabeled CS axon arbors caudal to the lesion were also represented. However, these data were described in a recent report (Freund et al., 2006) and will therefore not be considered further. At first sight, numerous BDAlabeled CS axon arbors are present in the gray matter rostral to the lesion (Fig. 4A-D), without obvious differences between the two groups of monkeys. To quantify the density of CS axon arbors in the gray matter rostral to the lesion, their cumulative length was measured (see Materials and Methods). The cumulative axon arbor length was then normalized by dividing by the total number of BDAlabeled CS axons present at C5 level in the white matter and plotted as a function of the extent (in percentage of the hemicord) of the lesion (Fig. 4E). If one excludes the animal Mk-CP (asterisk in Fig. 4E), which had an incomplete lesion of the CS tract, there is a clear trend showing that anti-Nogo-A antibody treated monkeys (red squares in Fig. 4E) displayed a higher accumulated axon arbor length than the control antibody treated monkeys (blue circles), thus indicating that the anti-Nogo-A antibody treatment enhanced axon sprouting in the gray matter rostral to the cervical lesion. However, this trend was not statistically significant (Mann and Whitney test, $P>$ 0.05).

More labeled CS axon arbors are visible rostral and caudal to the lesion in Mk-CG (control antibody treated) than in Mk-AC (anti-Nogo-A antibody treated); this apparent contradiction is due to the much higher (fourfold) total number of CS axons labeled with BDA in Mk-CG than in Mk-AC (see Table 1). If normalized according to the total number of CS axons labeled with BDA, then Mk-AC exhibits a larger cumulative axon arbor length rostral to the lesion (Fig. 4E) and a higher number of axon swellings caudal to the lesion (Fig. 4F) than Mk-CG (see also Freund et al., 2006). Furthermore, the extent of the lesion was smaller in Mk-CG than in Mk-AC.

CS axon arbors entering the lesion. On the parasagittal reconstructed sections used for the CS axon arbor length measurements, special attention was paid to CS axon arbors entering the scar tissue of the lesion. Examples are shown in Figure 5A, where more CS axons entered the lesion in three anti-Nogo-A antibody treated monkeys (red) than in one control antibody treated monkey (blue). For quantification, BDA-labeled CS axon arbors that crossed the rostral border of the lesion were counted in each monkey, normalized (according to the total number of BDA-stained CS axons at C5), and compared between the two groups of monkeys (Fig. 5D). All anti-Nogo-A antibody treated animals exhibited a higher number of CS axon arbors entering the scar tissue than the highest number of such axons found in the group of 
control monkeys (Fig. 5D, Table 1). This difference between the two groups of monkeys is statistically significant (Mann and Whitney test, $P<0.05$ ). Typically, the course of these fibers was highly irregular, showing that they were regenerating rather than surviving fibers. Nevertheless, even in anti-Nogo-A antibody treated monkeys, the number of CS axons entering the lesion remains very low (Fig. 5D). Furthermore, none of these CS fibers reached the caudal border of the lesion. These results indicate that the CS axon arbors observed caudal to the lesion (Freund et al., 2006) did not arise from transected CS axons, which would have regenerated straight through the lesion but rather from regenerating axons having grown around the lesion on bridges of spared white and gray matter.

CS axon collaterals entering the gray matter rostral to the cervical lesion. To determine whether antiNogo-A antibody treatment possibly enhanced CS axon collateral sprouting, the number of axon arbors leaving the cervical white matter and entering the gray matter was counted in the two groups of monkeys. Axons were counted within a territory extending as far as $3 \mathrm{~mm}$ rostral from the lesion (Figs. 1A, 5B). Only six monkeys (Mk-CB, Mk-CH, Mk-CP, Mk-AP, Mk-AM, and Mk-AG) fulfilled the criterion of sufficient quality of BDA staining to identify clearly such CS axon arbors leaving the white matter and entering the gray matter. The number of such CS axon arbors was normalized and plotted as a function of the extent of the hemicord lesion (Fig. 5E). The number of CS axon arbors passing from the white to the gray matter was lower in the three control antibody treated monkeys than in the three anti-Nogo-A antibody treated monkeys, without any obvious relationship to lesion size in either group of monkeys (Fig. 5E). For comparison, the same measurement was made in an intact monkey (Mk-I3; Table 2), exhibiting a normalized number of CS axon arbors entering the gray matter rostral to the lesion slightly lower than that for the three control antibody treated monkeys subjected to cervical cord lesion (Fig. 5E).

CS axons crossing the midline at $\boldsymbol{C 5}$. In the case of complete hemisection of the spinal cord, CS axons growing around the lesion in an attempt to reinnervate the denervated spinal cord caudal to the lesion would have to cross the spinal cord midline. For this reason, we examined the frontal sections taken at level C5 to determine whether BDA-labeled CS axons crossed the midline in the gray matter and whether such midline crossing was enhanced by the anti-Nogo-A antibody treatment (Fig. 5C; see also Materials and Methods). The sum of CS axons crossing the midline at C5 in each monkey was normalized to the total number of labeled CS axons and plotted as a function of the lesion extent (Fig. 5F). The anti-Nogo-A antibody treated monkeys exhibited a higher number of midline crossing CS axons than the control antibody treated monkeys (Fig. 5F). There was no relationship to the lesion extent in either group of monkeys. For comparison, the same analysis was conducted in three intact monkeys (Table 2), exhibiting a normalized number of CS axons crossing the midline at C5 equal or lower than the four control antibody treated monkeys subjected to cervical cord lesion (Fig. 5F).

\section{DISCUSSION}

The present study provides evidence that, after unilateral cervical lesion that sections the dorsolateral funiculus, anti-Nogo-A antibody treatment decreases the normally occurring retrograde degeneration of the CS axotomized axons: the number of terminal retraction bulbs formed by the axotomized CS axons was smaller in the group of anti-Nogo-A antibody treated monkeys than in the group of control antibody treated monkeys (Fig. 2D), and the terminal retraction bulbs were closer to the lesion (Fig. 2E,F). Thus, anti-Nogo-A antibody treatment prevented to some extent the phenomenon of axonal dieback. More CS axons grew into the lesion scar, but their regeneration through the scar tissue remained unsuccessful. In contrast, increased numbers of CS axon arbors were seen in the anti-Nogo-A antibody treated spinal cord rostral to the lesion. Some of these fibers arborized in the spinal cord caudal to the injury, as recently reported (Freund et al., 2006).

With respect to the question of direct regeneration of the cut stem axon or collateral axonal sprouting, there is evidence here that both were enhanced by anti-Nogo-A antibody treatment. Axotomized CS axons growing and penetrating into the lesion area were more numerous in the anti-Nogo-A antibody treated monkeys than in the control antibody treated ones. However, none of the CS axons penetrating the scar seemed to be able to reach its distal end. Scar-associated growth inhibition factors probably account for this blockade of regeneration (Rhodes and Fawcett, 2004).

CS axons rostral to the injury gave rise to collaterals. The anti-Nogo-A antibody treatment enhanced the presence of CS axon arbors in the gray matter rostral to the lesion, the number of CS axon arbors (possibly corresponding to CS axon collaterals) passing from the white to the gray matter, and finally the number of CS axon arbors crossing the segmental midline at the C5 level. These measurements show that axonal sprouting took place rostral to the cervical lesion, and that some of these fibers were in position to grow around the lesion in order to reinnervate the spinal cord territory caudal to the lesion deprived of CS input. The anti-Nogo-A antibody treatment enhanced CS axonal sprouting and regrowth, confirming previous observations in anti-Nogo-A antibody treated rats (Liebscher et al., 2005).

The larger number of CS axon arbors observed caudal to the lesion in the anti-Nogo-A antibody treated monkeys correlates with enhancement of functional recovery, especially of manual dexterity, i.e., functions known to depend on direct inputs from CS fibers (Freund et al., 2006). As discussed further below, in our view the present data suggest a mechanism of functional recovery relying on collateral sprouting that arises from the axotomized CS axons rostral to the lesion, possibly contributing to the increased presence of CS axonal arbors in the gray matter of the spinal segments caudal to the lesion (green curved lines in Fig. 1A). Again, these data are consistent with previous data in rodents showing that "regenerative sprouting" enhanced in anti-Nogo-A antibody treated rats consisted of CS collaterals growing around the lesion on remaining tissue bridges (see, e.g., Von Meyenburg et al., 1998; Brösamle et al., 2000; Schwab, 2004; Liebscher et al., 2005). 
The present study did not address the possibility that some CS axon arbors observed caudal to the lesion (Freund et al., 2006) could result from sprouting arising from the undecussated CS axons not affected by the cervical lesion (Fig. 1A). In rats subjected to a spinal cord lesion interrupting the main CS tract, axon sprouting from the intact ventral CS tract was indeed found to play an important role in the spontaneous postlesion recovery (Weidner et al., 2001). CS fibers from the intact side have been seen to cross the spinal cord midline in rats after unilateral pyramidotomy and anti-Nogo-A antibody application (Thallmair et al., 1998). In earlier studies in macaques, we found that reversible inactivation by intracortical muscimol infusion in the contralesional hemisphere several months postlesion leads to a loss of recovered manual dexterity (Schmidlin et al., 2004; see brown syringe in Fig. 1A). In contrast, reversible inactivation of the ipsilesional hemisphere in control antibody treated monkeys did not affect the postlesional functional recovery (Schmidlin et al., 2005), indicating that the decussated CS tract originating from the ipsilesional hemisphere does not play a major role in the recovery. Altogether, the present data as well as our recent report (Freund et al., 2006) favor the notion that collateral sprouting and growth of the axotomized CS tract after cervical cord lesion may play a role in the postlesion functional recovery.

It remains to be determined whether other descending tracts (rubrospinal, reticulospinal, long propriospinal projections) might also contribute, as shown in the rat (see, e.g., Hashimoto and Fukuda, 1991; Feraboli-Lohnherr et al., 1997; Raineteau et al., 2001; Fouad et al., 2001; Schucht et al., 2002; Ruitenberg et al., 2003; Bareyre et al., 2004). The possibility that the rubrospinal and reticulospinal tracts may play a role in the functional recovery is consistent with the observation reported by Freund et al. (2006) that the two control antibody treated monkeys with a lesion leaving intact a significant portion of these two tracts spontaneously recovered much better than the control antibody treated animal in which the lesion interrupted these two tracts completely.

The correlation between the anti-Nogo-A antibody enhancement of CS sprouting rostral to the cervical lesion (present study) and the density of CS tract axons caudal to the lesion (Freund et al., 2006) is shown in Figure 4F. The normalized cumulative axon arbor length as determined rostral to the lesion (present study) was plotted as a function of the normalized number of CS axonal swellings found caudal to the lesion, as reported recently (Freund et al., 2006). The control antibody treated monkeys subjected to complete transection of the dorsolateral funiculus exhibited no or only few CS axonal swellings caudal to the lesion; this was correlated with limited axonal sprouting rostral to the lesion in these animals (Fig. 4F, three blue circles on the left of the plot). In sharp contrast, the four anti-Nogo-A antibody treated monkeys, all subjected to complete transection of the dorsolateral funiculus, exhibited much higher numbers of CS axonal swellings caudal to the lesion. This again correlated with a significantly higher density of CS axon arbors arising from sprouts rostral to the lesion (red squares in Fig. 4F). The control antibody treated monkey Mk-CP (asterisk in Fig. 4F) has to be considered separately, because the lesion of the dorsolateral funiculus was incomplete (Fig. 1C), leaving intact a small bundle of CS axons. The intact CS axons reaching the region caudal to the lesion sprouted profusely at that level, giving rise to numerous axon arbors and swellings. This animal showed a much better functional recovery than the other control antibody treated monkeys (Freund et al., 2006).

\section{COMPARISON WITH PREVIOUS STUDIES}

The present model of cervical cord lesion in the monkey can be compared, at least from a behavioral point of view, with previous studies in which macaque monkeys were subjected to a lesion of the cervical cord (Galea and Darian-Smith, 1997; Sasaki et al., 2004). Although the transection of the dorsolateral funiculus was aimed at lower cervical segments $(\mathrm{C} 7 / \mathrm{C} 8)$ than in these two studies (C3-C4), we observed an extent and a time course of functional recovery (Freund et al., 2006) largely comparable to that reported by Galea and Darian-Smith (1997). In contrast, Sasaki et al. (2004) reported an even faster, if not immediate, time course of recovery of precision grip and independent finger movements (1-28 days), although weakness in force and deficit in preshaping lasted longer. The two studies of lesions at the C3-C4 level (Galea and Darian-Smith, 1997; Sasaki et al., 2004) did not analyze the plasticity of the CS tract in the vicinity of the cervical lesion, thus preventing a comparison with the present anatomical data.

A different neutralizing antibody against Nogo-A, mAbIN-1, was applied in marmosets with a thoracic lesion (Fouad et al., 2004). CS fibers growing around the lesion
Fig. 4. A-D: Superimposed reconstructions of parasagittal sections of the cervical-thoracic cord showing the lesion (vertical arrow) as well as the density of BDA-labelled CS axon arbors present around the lesion, in four different monkeys (with a reminder of their lesion extent). Two monkeys received the control antibody (Mk-CH and Mk-CG in A and C), whereas in the other two monkeys the antiNogo-A antibody was delivered (Mk-AM and Mk-AC in B and D). Rostral to the lesion, the densely packed brown line segments represent the BDA-labelled CS axons in the white matter, interrupted by the transection of the dorsolateral funiculus. The position of the rostral stump of CS fibers in the white matter and of the axon arbors in the gray matter is schematic, as derived from a superimposition of sections, some of which were located more lateral than those for which their contour is represented here. The black line segments represent the CS axon arbors traced rostrally in the gray matter and caudally to lesion. In these four monkeys, the dorsolateral funiculus was completely transected. A less extended version of these four reconstruc- tions has been previously published by Freund et al. (2006), which contained at that time only the axon arbors in the gray matter caudal to the lesion. Here, the reconstructions have been extended to represent also the CS axon arbors rostral to the lesion, which are the topic of the present report. E: Relationship between the normalized cumulated axon arbor length in the gray matter rostral to the lesion as a function of the extent (in percentage) of the hemicord lesion. Cumulated axon arbor lengths were measured only rostral to the lesion. Blue circles are for control antibody treated monkeys, whereas red squares are for the anti-Nogo-A antibody treated monkeys. The site of this analysis is schematically represented by the number 3 in Figure 1A. F: The present data on the axonal sprouting of the CS tract rostral to the lesion (reflected here by the normalized cumulated axon arbor length) were plotted as function of the normalized number of CS axonal swellings observed caudal to the lesion, as reported recently (Freund et al., 2006) 

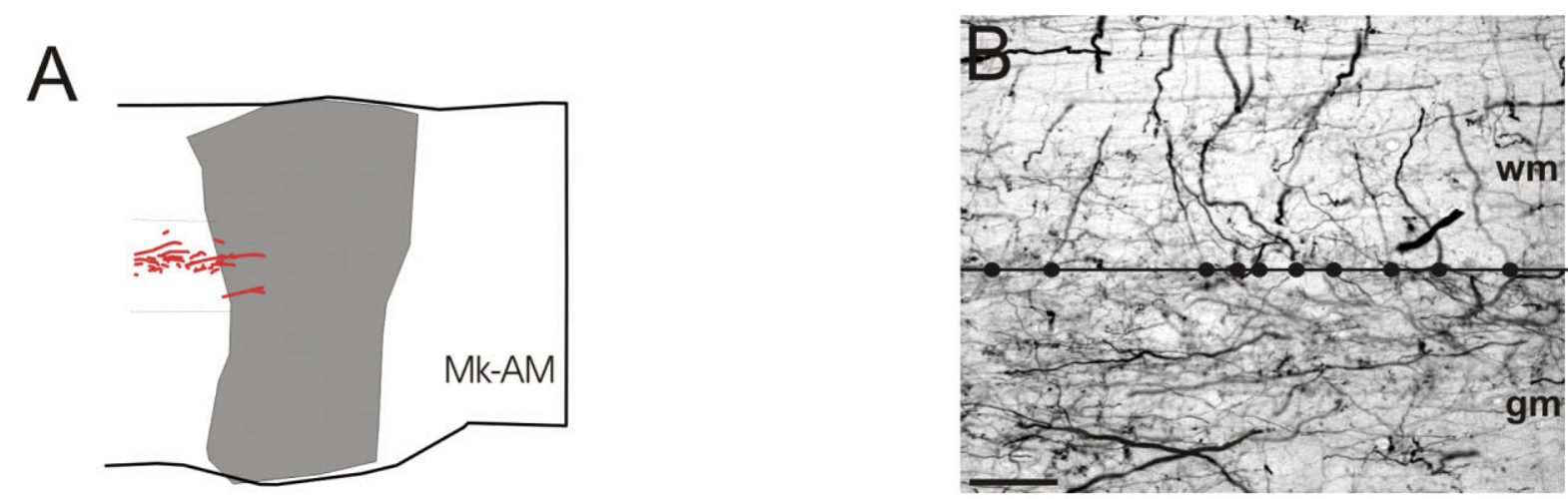

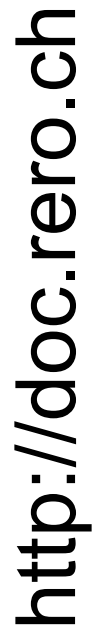
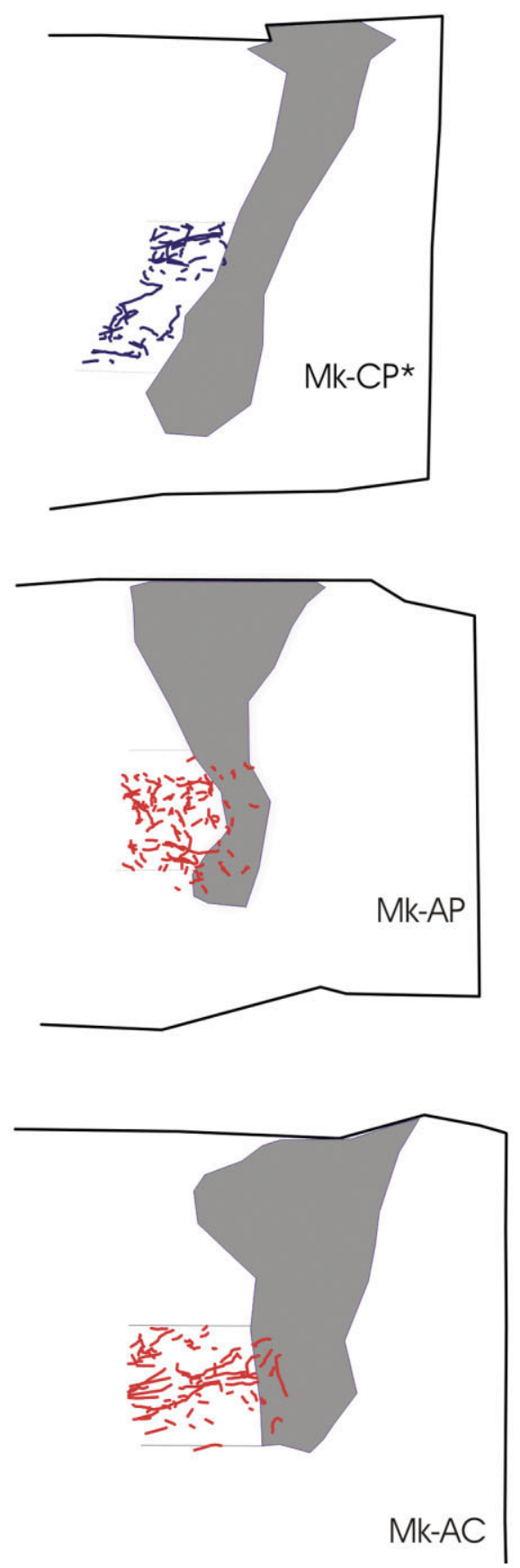

D
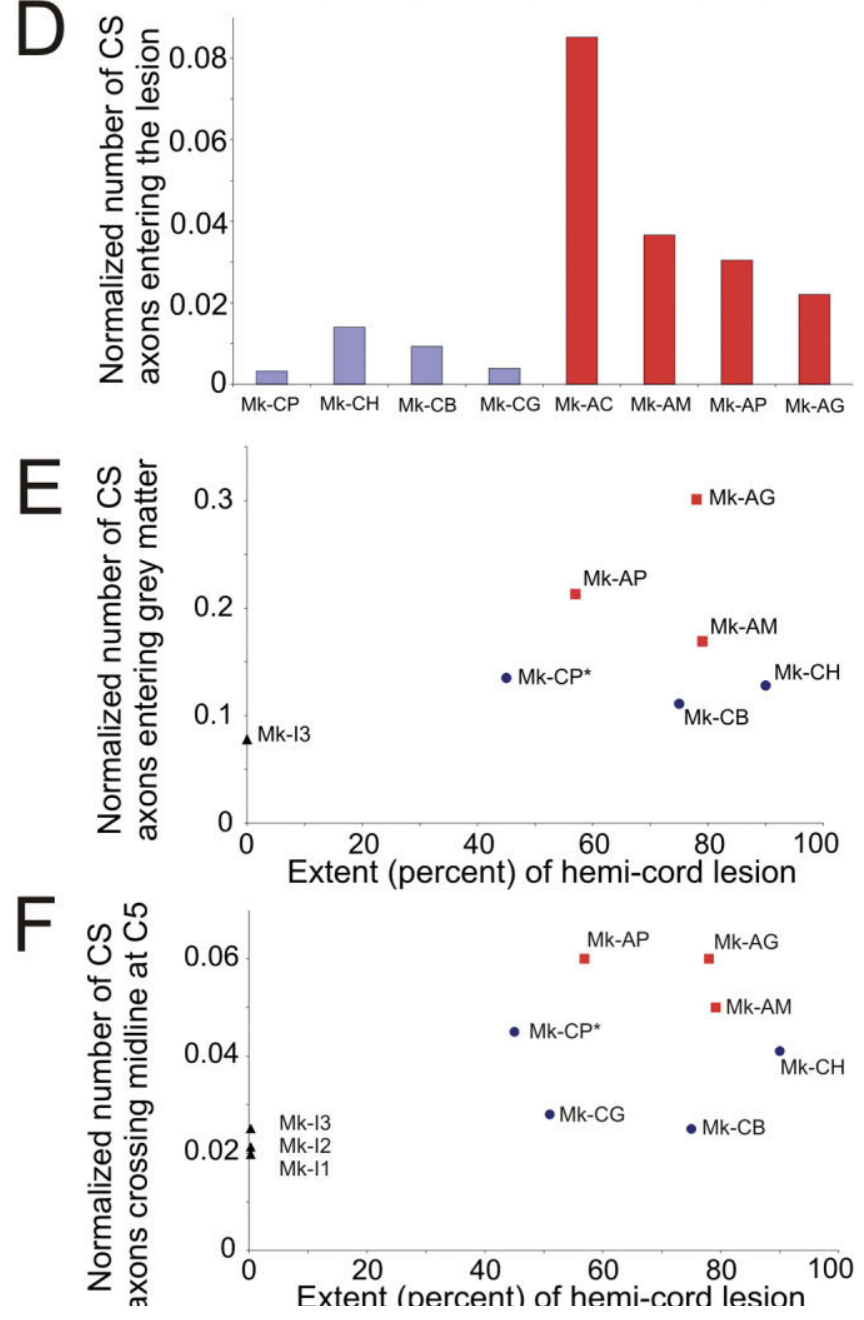
TABLE 2. List of the Three "Intact" Monkeys Included in the Present Study With Identification Code ${ }^{1}$

\begin{tabular}{|c|c|c|c|}
\hline & Mk-I1 & Mk-I2 & Mk-I3 \\
\hline Species & Fasc. & Fasc. & Fasc. \\
\hline Age at death (years); weight (kg) & $4 ; \sim 5$ & $8.3 ; \sim 10$ & $7.7 ; \sim 10$ \\
\hline Volume of BDA injected (in $\mu \mathrm{l}$ ) & 10 & 22.5 & 25.5 \\
\hline No. of injection sites & 7 & 15 & 17 \\
\hline No. of injection tracks & 3 & 9 & 11 \\
\hline Survival time postinjection (days) & 25 & 42 & 48 \\
\hline $\begin{array}{l}\text { Normalized No. of CS axons going from } \\
\text { white to grey matter }\end{array}$ & $-^{2}$ & $-^{3}$ & 0.078 \\
\hline $\begin{array}{l}\text { Normalized No. of CS axons crossing } \\
\text { midline at C5 }\end{array}$ & 0.02 & 0.021 & 0.025 \\
\hline $\begin{array}{l}\text { No. of CS axons in the white matter at C5 } \\
\text { (for normalization) }\end{array}$ & 1,766 & 984 & 1,922 \\
\hline No. of crossed CS axons at C5 (\% of total) & $1,620(91.7)$ & $859(87.3)$ & $1,735(90.3)$ \\
\hline No. of uncrossed CS axons at C5 (\% of & $146(8.3)$ & $125(12.7)$ & $187(9.7)$ \\
\hline
\end{tabular}

total)

${ }^{1}$ The data in italics derived from these three intact (unlesioned, untreated) animal were used to establish a baseline for the analyses presented for the lesioned represented monkeys in Figure 5E,F. In the two intact monkeys (Mk-I2 and Mk-I3), injection of tracer into the motor cortex was the unique intervention, following the protoco described in Materials and Methods. Mk-I1 was used in a previous tracing study (Rouiller et al., 1996), in which the primary motor cortex was mapped by using intracortical microstimulation before BDA injection. Under species, Fasc. is for Macaca fascicularis. ${ }^{2} \mathrm{In} \mathrm{Mk}-\mathrm{I} 1$, the cervical segment C6-T3 was not cut in the sagittal plane (the matter could not be made. ${ }^{3} \mathrm{In} \mathrm{Mk}-\mathrm{I} 2$, the quality of the BDA reaction at C6-T3 did not allow the assessment of the number of axons entering the gray matter. In contrast, the block including C5 (sectioned in the frontal plane) was processed separately, and the BDA reaction allowed the determination of the number of CS axons crossing midline.

and into the caudal spinal cord were observed (Fouad et al., 2004), but the animals were not tested behaviorally, preventing correlation with functional recovery.

A postlesion sprouting of the CS tract was shown to be present in hamster only at perinatal ages (Kuang and Kalil, 1990). Adult hamsters subjected to pyramidotomy exhibited a retraction of the axons rostral to the lesion by a substantial distance, up to about 6-7 $\mathrm{mm}$ after several months. The present data, derived from macaque monkeys subjected to cervical lesion, are markedly different because the distance of retraction was much smaller. Indeed, after 4-6 months postinjury, most terminal retraction bulbs were located not more than $1 \mathrm{~mm}$ from the lesion. Furthermore, in the marmoset as well, the terminal retraction bulbs appeared to be located close to the lesion after 2 weeks postinjury (Fouad et al., 2004). These data are in line with earlier reports in monkey and man that retrograde degeneration of axotomized CS axons was limited to the region immediately above the lesion (Tower,
1940; Bronson et al., 1978). In rats, axonal dieback following spinal cord lesion was studied for the rubrospinal and reticulospinal tracts (Houle and Jin, 2001). The authors observed an average retraction of the axons over a mean distance of about $500 \mu \mathrm{m}$, which is of the same order as the retraction of the CS axons seen in monkeys (Fouad et al., 2004; present study). The limited axonal dieback is thus favorable for a regenerative sprouting from the axotomized axons, giving rise to collaterals that can grow in the caudal direction around the lesion in order to reinnervate the region caudal to the lesion.

In the present study, young adult monkeys (3.5-6.9 years old at time of death) were subjected to spinal cord lesion (Table 1). It cannot be excluded that some of the axonal sprouting (although limited) observed in the control antibody treated monkeys occurred because of the relatively juvenile state of some of the animals and would not have taken place in older animals. Similarly, it is not established whether the enhancement of sprouting resulting from the anti-Nogo-A treatment would have reached the same extent in older animals. Nevertheless, most of our animals (except maybe Mk-AG) had an age at the time of the lesion (3.5 years or more) by which the myelination of the CS tract reached the adult stage, insofar as full myelination was reported to last for as long as 36 months (Olivier et al., 1997). As far as the CS tract is concerned, the animals included in the present study can thus be considered as adult monkeys.

As a result of BDA injection in M1, the number of CS-labeled axons observed at the C5 level on transverse sections (up to 2,282; see Table 1) appears low considering that the total number of CS axons was estimated to be 400,000 and that the number of CS neurons retrogradely labeled after tracer injection in the upper spinal cord amounted to 72,000 in the entire frontal lobe (Dum and Strick, 1991). There are a number of possible explanations for this result. First, only 50\% of the precentral CS axons originate from M1 (Dum and Strick, 1991; He et al., 1993, 1995). Second, the BDA injections formed patchy territories, leaving in-between zones where there was no or less uptake. Third, the BDA injections did not cover the entire M1. Fourth, a contingent of CS axons terminates above C5. In addition, BDA infusion was limited to the rostral bank of the central sulcus; therefore, the whole rostral part of M1 was not injected. Furthermore, compared with
Fig. 5. A: Reconstructions of one parasagittal section of the cervical-thoracic cord for each monkey, showing the lesion at C7/C8 (gray area) as well as the BDA-labeled CS axon arbors present rostral to the lesion (up to a distance of $500 \mu \mathrm{m}$ from the lesion) in four monkeys. For three anti-Nogo-A antibody treated monkeys (Mk-AM, Mk-AP, Mk-AC), the CS axon arbors were drawn in red, whereas, for one control antibody treated monkey, they were drawn in blue. B: Photomicrograph of a parasagittal cervical cord, showing the region rostral to the lesion where numerous BDA-labeled CS axons and axon collaterals are present in the white and gray matter. The black line delineates the border between gray and white matter. CS axon arbors crossing this line (mainly in a perpendicular orientation) were counted and normalized by dividing their cumulative sum across sections by the total number of CS axons labeled at C5 level. C: Photomicrograph of a cervical spinal cord frontal section taken at the C5 level showing at higher magnification the central part of the cervical cord, with some CS axons crossing the midline and terminating in the opposite gray matter. CS collaterals intercepting one of black vertical lines were counted, and the cumulative number was normalized by dividing it by the total number of labeled CS fibers. D: Normalized number of BDA-labeled CS axon arbors crossing the rostral border of the lesion in order to enter into the lesion area. The site of this analysis is schematically represented by the number 4 in Figure 1A. E: The normalized cumulated number of CS axon arbors crossing the white/gray matter border along an axis perpendicular to this border (see Fig. 5B) was plotted as a function of the extent of the hemicord lesion (in percentage). Blue circles are for control antibody treated monkeys, whereas anti-Nogo-A antibody treated monkeys are represented by a red square. Data derived from an intact monkey are shown by a black triangle. The site of this analysis is schematically represented by the number 5 in Figure 1A. F: The normalized number of CS axon collaterals crossing the midline at the C5 level (see Fig. 5C) was plotted as a function of the extent of the hemicord lesion (in percentage). Blue circles are for control antibody treated monkeys, whereas anti-Nogo-A antibody treated monkeys are represented by a red square. Data derived from three intact monkeys are shown by black triangles. The site of this analysis is schematically represented by the number 6 in Figure 1A. Scale bars $=50 \mu \mathrm{m}$ in B; $100 \mu \mathrm{m}$ in C. 
uptake of other anterograde tracers, such as WGA-HRP, the uptake of BDA appears less prominent. However, to explain the surprisingly low number of CS axons labeled with BDA in the present study, one may consider the likely possibility that the very fine axons making up the majority of the CS tract are not labeled or escaped detection at the light microscopic level, as suggested by our observation that most BDA-labeled CS axons visible in our material at the C5 level are relatively large in diameter. In this context, for the same reason, one cannot exclude that some fine CS axons unlabeled with BDA or undetected at the light microscopic level were spared by the lesion.

In conclusion, the present study demonstrates quantitatively that anti-Nogo-A antibody treatment attenuated the retraction of axotomized CS axons after cervical cord injury and enhanced axonal sprouting rostral to the lesion. Within the scar tissue, growth of these fibers is blocked by inhibitory factors other than Nogo-A. Some fibers, however, succeeded in bypassing the scar, presumably by remaining on gray matter bridges. These axons growing around the lesion probably make an important contribution to the incomplete but significantly enhanced reconstruction of the CS fiber plexus caudal to the lesion in the anti-Nogo-A antibody treated monkeys (Freund et al., 2006), moreso than the fibers attempting to regenerate and to reenter the lesion itself. The contingent of CS axons growing around the lesion in the gray matter is believed to be an anatomical correlate of the functional recovery observed in the anti-Nogo-A antibody treated animals.

\section{ACKNOWLEDGMENTS}

The authors thank Dr. Alexandre Babalian and Juliane Aebischer for the acquisition and interpretation of some data. The authors are grateful for the technical assistance of Georgette Fischer, Véronique Moret, Françoise Tinguely, Christiane Marti, Monika Bennefeld, and Christine Roulin (histology and behavioral evaluations); Josef Corpataux, Bernard Bapst, Laurent Bossy, and Bernard Morandi (animal housekeeping); André Gaillard (mechanics); Bernard Aebischer (electronics); and Laurent Monney (informatics). The authors also thank Dr. Clive Brown for proofreading the revised version of the manuscript.

\section{LITERATURE CITED}

Bareyre FM, Kerschensteiner M, Raineteau O, Mettenleiter TC, Wein mann O, Schwab ME. 2004. The injured spinal cord spontaneously forms a new intraspinal circuit in adult rats. Nat Neurosci 7:269-277.

Bregman BS, Kunkel-Bagden E, Schnell L, Dai HN, Gao D, Schwab ME. 1995. Recovery from spinal cord injury mediated by antibodies to neurite growth inhibitors. Nature 378:498-501.

Bronson R, Gilles FH, Hall J, Hedley-Whyte ET. 1978. Long term posttraumatic retrograde corticospinal degeneration in man. Hum Pathol 9:602-607

Brösamle C, Huber AB, Fiedler M, Skerra A, Schwab ME. 2000. Regeneration of lesioned corticospinal tract fibers in the adult rat induced by a recombinant, humanized IN-1 antibody fragment. J Neurosci 20:80618068.

Caroni P, Savio T, Schwab ME. 1988. Central nervous system regeneration: oligodendrocytes and myelin as non-permissive substrates for neurite growth. Prog Brain Res 78:363-370.

Dum RP, Strick PL. 1991. The origin of corticospinal projections from the premotor areas in the frontal lobe. J Neurosci 11:667-689.

Feraboli-Lohnherr D, Orsal D, Yakovleff A, Ribotta MGY, Privat A. 1997. Recovery of locomotor activity in the adult chronic spinal rat after sublesional transplantation of embryonic nervous cells: specific role of serotonergic neurons. Exp Brain Res 113:443-454.

Fouad K, Volker D, Schwab ME. 2001. Improving axonal growth and functional recovery after experimental spinal cord injury by neutralizing myelin associated inhibitors. Brain Res Rev 36:204-212.

Fouad K, Klusman I, Schwab ME. 2004. Regenerating corticospinal fibers in the marmoset (Callitrix jacchus) after spinal cord lesion and treatment with the anti-Nogo-A antibody IN-1. Eur J Neurosci 20:24792482 .

Freund P, Schmidlin E, Wannier T, Bloch J, Mir A, Schwab ME, Rouiller EM. 2006. Nogo-A-specific antibody treatment enhances sprouting and functional recovery after cervical lesion in adult primates. Nat Med $12: 790-792$

Galea MP, Darian-Smith I. 1997. Manual dexterity and corticospinal connectivity following unilateral section of the cervical spinal cord in the macaque monkey. J Comp Neurol 381:307-319.

Hashimoto T, Fukuda N. 1991. Contribution of serotonin neurons to the functional recovery after spinal cord injury in rats. Brain Res 539:263270.

He SQ, Dum RP, Strick PL. 1993. Topographic organization of corticospinal projections from the frontal lobe: motor areas on the lateral surface of the hemisphere. J. Neurosci 13:952-980.

He SQ, Dum RP, Strick PL. 1995. Topographic organization of corticospinal projections from the frontal lobe: motor areas on the medial surface of the hemisphere. J Neurosci 15:3284-3306.

Holmes GL, May WP. 1909. On the exact origin of the pyramidal tracts in man and other mammals. Brain 32:1-42.

Houle JD, Jin Y. 2001. Chronically injured supraspinal neurons exhibit only modest axonal dieback in response to a cervical hemisection lesion. Exp Neurol 169:208-217.

Jenny AB, Inukai J. 1983. Principles of motor organization of the monkey cervical spinal cord. J Neurosci 3:567-575.

Kalil K, Schneider GE. 1975. Motor performance following unilateral pyramidal tract lesions in the hamster. Brain Res 100:170-174.

Kao CC, Chang LW, Bloodworth JMB Jr. 1977a. Electron microscopic observations of the mechanisms of terminal club formation in transected spinal axons. J Neuropathol Exp Neurol 36:140-156.

Kao CC, Chang LW, Bloodworth JMB Jr. 1977b. The mechanisms of spinal cord cavitation following spinal cord transection. J Neurosurg 46:745756 .

Kerschensteiner M, Schwab ME, Lichtman JW, Misgeld T. 2005. In vivo imaging of axonal degeneration and regeneration in the injured spinal cord. Nat Med 11:572-577.

Kuang RZ, Kalil K. 1990. Specificity of corticospinal axon arbors sprouting into denervated contralateral spinal cord. J Comp Neurol 302:461-472.

Lacroix S, Havton LA, McKay H, Yang H, Brant A, Roberts J, Tuszynski MH. 2004. Bilateral corticospinal projections arise from each motor cortex in the macaque monkey: a quantitative study. J Comp Neurol 473:147-161.

Lassek AM. 1942. The pyramidal tract. A study of retrograde degeneration in the monkey. Arch Neurol 48:561-567.

Levin PM, Bradford FK. 1938. The exact origin of the cortico-spinal tract in the monkey. J Comp Neurol 68:411-422.

Liebscher T, Schnell L, Schnell D, Scholl J, Schneider R, Gullo M, Fouad K, Mir A, Rausch M, Kindler D, Hamers FPT, Schwab ME. 2005. Nogo-A antibody improves regeneration and locomotion of spinal cord-injured rats. Ann Neurol 58:706-719.

Oertle T, Van der Haar ME, Bandtlow CE, Robeva A, Burfeind P, Buss A, Huber AB, Simonen M, Schnell L, Brösamle C, Kaupmann K, Vallon R, Schwab ME. 2003. Nogo-A inhibits neurite outgrowth and cell spreading with three discrete regions. J Neurosci 23:5393-5406.

Olivier E, Edgley SA, Armand J, Lemon R. 1997. An electrophysiological study of the postnatal development of the corticiospinal system in the macaque monkey. J Neurosci 17:267-276.

Pernet U, Hepp-Reymond M-C. 1975. Retrograde Degeneration der Pyramidenbahnzellen im motorischen Kortex beim Affen (Macaca fascicularis). Acta Anat 552-561.

Raineteau O, Fouad K, Noth P, Thallmair M, Schwab ME. 2001. Functional switch between motor tracts in the presence of the mAb IN-1 in the adult rat. Proc Natl Acad Sci U S A 98:6929-6934.

Rhodes KE, Fawcett JW. 2004. Chondroitin sulphate proteoglycans:preventing plasticity or protecting the CNS? J Anat 204:33-48.

Rouiller EM, Moret V, Tanné J, Boussaoud D. 1996. Evidence for direct connections between the hand region of the supplementary motor area 
and cervical motoneurons in the macaque monkey. Eur J Neurosci 8:1055-1059.

Rouiller EM, Tanné J, Moret V, Kermadi I, Boussaoud D, Welker E. 1998 Dual morphology and topography of the corticothalamic terminals originating from the primary, supplementary motor, and dorsal premotor cortical areas in macaque monkeys. J Comp Neurol 396:169-185.

Ruitenberg MJ, Plant GW, Hamers FPT, Wortel J, Blits B, Dijkhuizen PA, Gispen WH, Boer GJ, Verhaagen J. 2003. Ex vivo adenoviral vectormediated neurotrophin gene transfer to olfactory ensheathing glia: effects on rubrospinal tract regeneration, lesion size, and functional recovery after implantation in the injured rat spinal cord. J Neurosci 23:7045-7058

Sasaki S, Isa T, Pettersson LG, Alstermark B, Naito K, Yoshimura K, Seki K, Ohki Y. 2004. Dexterous finger movements in primate without monosynaptic corticomotoneuronal excitation. J Neurophysiol 92: 3142-3147.

Schmidlin E, Wannier T, Bloch J, Rouiller EM. 2004. Progressive plastic changes in the hand representation of the primary motor cortex parallel incomplete recovery from a unilateral section of the corticospinal tract at cervical level in monkeys. Brain Res 1017:172-183.

Schmidlin E, Wannier T, Bloch J, Belhaj-Saif A, Wyss A, Rouiller EM 2005. Reduction of the hand representation in the ipsilateral primary motor cortex following unilateral section of the corticospinal tract at cervical level in monkeys. BMC Neurosci 6:56.

Schnell L, Schwab ME. 1990. Axonal regeneration in the rat spinal cord produced by an antibody against myelin-associated neurite growth inhibitors. Nature 343:269-272.
Schucht P, Raineteau O, Schwab ME, Fouad K. 2002. Anatomical correlates of locomotor recovery following dorsal and ventral lesions of the rat spinal cord. Exp Neurol 176:143-153.

Schwab ME. 2004. Nogo and axon regeneration. Curr Opin Neurobiol 14:118-124.

Thallmair M, Metz GAS, Z'Graggen WJ, Raineteau O, Kartje GL, Schwab ME. 1998. Neurite growth inhibitors restrict plasticity and functional recovery following corticospinal tract lesions. Nat Neurosci 1:124-131.

Tower SS. 1940. Pyramidal lesion in the monkey. Brain 63:36-90.

Von Meyenburg J, Brösamle C, Metz GAS, Schwab ME. 1998. Regeneration and sprouting of chronically injured corticospinal tract fibers in adult rats promoted by NT-3 and the mAb IN-1, which neutralizes myelin-associated neurite growth inhibitors. Exp Neurol 154:583-594.

Wannier T, Schmidlin E, Bloch J, Rouiller EM. 2005. A unilateral section of the corticospinal tract at cervical level in primate does not lead to measurable cell loss in motor cortex. J Neurotrauma 22:703-717.

Weidner N, Ner A, Salimi N, Tuszynski MH. 2001. Spontaneous corticospinal axonal plasticity and functional recovery after adult central nervous system injury. Proc Natl Acad Sci U S A 98:3513-3518.

Weinmann O, Schnell L, Ghosh A, Montani L, Wiessner C, Wannier T, Rouiller E, Mir A, Schwab ME. 2006. Intrathecally infused antibodies against Nogo-A penetrate the CNS and downregulate the endogenous neurite growth inhibitor Nogo-A. Mol Cell Neurosci 32:161-173.

Wohlfarth S. 1932. Die vordere Zentralwindung bei Pyramidenbahnläsionen verschiedener Art. Eine histopathologische Untersuchung. Acta Med Scand Suppl 46:1-235. 